\title{
Agricultural Research Resource Sharing Model Based Grids
}

\author{
Liu Haihui
}

\author{
College of Computer and Communication Engineering, Weifang University, Weifang, China
}

\begin{abstract}
Grids provide a high-performance computing, resource management and service capacity through the composition of high-speed Internet, fully integrated sharing resources including geographically distributed computing resources such as CPU, memory, databases, etc. There exist duplication in the system, some devices are frequently used, and some devices are long idle, there is ineffective share in China's agricultural scientific research and resources, so the agricultural research and resource sharing platform based on Grid are constructed. making the network's each user can access shared services with other users authorization, can solve "information isolated island" problem which exists in agricultural informationization to a certain extent, effectively use the resources of agricultural research to promote our country's rapid development in agricultural science research.
\end{abstract}

Index Terms: Agricultural; Research; Agricultural Informationization; Grids; Resource Sharing; Network

(C) 2011 Published by MECS Publisher. Selection and/or peer review under responsibility of the International Conference on E-Business System and Education Technology

\section{Introduction}

Today, scientific and technological innovation has become the main driving force of economic development, high-tech such as biotechnology, information technology, are widely used in agriculture, completely changed the traditional small-scale agricultural production and management, the World Agriculture changed from "resource dependent type" to "technology dependent type ", " Information dependent type ". It indicates a huge challenge to our country whose main agricultural model is "resource dependent type".

With the rapid development of modern science and technology, strengthening research and innovation has become a consensus for all walks of life. For agricultural research institutions, research resources influence and determine the degree of scientific research and progress in agriculture to a certain extent, experimental means quality and level influence and determine the scale of agricultural science experiments and transformation of scientific research. China has abundant agricultural research resources. According to an estimate, about several hundred million dollars of investment in agricultural research resources do not achieve the desired results. The main reason is that the information about agriculture research cannot communicate well, are limited by physical

\footnotetext{
* Corresponding author.

E-mail address: wflhh@sohu.com
} 
distance; there have been idle resources and facilities in one place while the other place lacks the necessary research equipment [1] [2].

Even though some research groups can use these resources through some people, they must run from one place to another, it is time-consuming and laborious. In addition, with the rapid development of science and technology, labor are divided more and more detail, An organization are difficult to undertake a larger research and development projects independently, there is growing need for a distributed collaborative research and resource sharing and collaboration environment. How to achieve the effective sharing of agricultural research resources, how to ensure reliable and timely service, how to achieve remote operation of agricultural resources have become an urgent research topic.

Agricultural resources sharing is to provide a resources sharing platform in a certain range using of information technology, we are free to enter or exit the resources on this platform, using a variety of platform management features to get agricultural research resources sharing; which is characterized by digital, network, intelligence, and multimedia, and its basic features is an open, sharing, interaction, collaboration [1]. Agricultural research and resources need to improve their efficiency and reduce financial, material and labor costs; need to achieve their interconnection and interoperability, and enhance their capacity to better serve the agricultural research services; to achieve this we must realize agricultural research and resource sharing.

Grids enables computing resources, storage resources, data resources, information resources, knowledge resources; experts' resource to share fully conveniently, the agricultural resources sharing based on grids can effectively alleviate the current difficulties[3][4][5].

\section{Grids}

Following the traditional Internet, Web, grids are the third wave of information revolution, to be called thirdgeneration Internet. It is a new kind of technology, changing and developing all the time [6] [7]. Then there is no exact definition of the grids. To be simple, grids is the basic network infrastructure in this information society. It can share all the resources including computing resources, storage resources, correspondence resources, software resources, information resources, and knowledge resources and so on[6].

The grids will connect all the information and the isolated resources, and will let people's work and lives more convenient.

First, the grids can share resources, it can eliminate the isolated information, make the application procedures connect to each other. The grids and the computer network are different, the computer network makes the hardware connect, but the grids can make the application layer connect. Next, the second characteristic is the joint operation; many grids nodes may process a project together. Third, the grids is based on the international open technical standard, so this is different from many professions, departments or the software promoted by the company. Finally, the grids may provide the dynamic service, which adapts the change [8].

Grids combines high performance computer, data sources, Internet together. Compared with Internet, it has the following benefits: high performance, integration, knowledge production, resources sharing.

The high performance of the grids means that is has a stronger function and performance compared to Internet. It reveals in four aspects.

First, the grids internet has a greater bandwidth compared to Internet. Europe's and America's grids plan can use the high velocity branch net.

Second, in the grids there will have more high performances computers, therefore the grids computation speed; the data processing speed will be enhanced in a large scale.

Third, the grids system structure will be able to use these resources more effectively than Internet. For instance, the grids will use a kind of technology called "wide area buffer technology"; it automatically put information which users need most on the nearest server to the user.

Fourth, the grids will promote much more and bigger on-line communities' appearance. This mutually joined community will finally constitute a huge grids community, which makes all citizens on our Earth become an entire entity. Grids security mechanisms meet the security various requirements better, such as data encryption, token authentication, to prevent the illegal invasion and illegal use.

Technical characteristics of agricultural research resource sharing are digital, networking, intelligent and multi-media, and its basic features are open, sharing, interaction and collaboration. The grids can provide just 
such an environment in which all kinds of equipment resources are open, sharing, interaction and collaboration, and the grids also solve real problems from this perspective of technology, so the grids for agricultural research resources equipment provide a new way to share.

The agricultural research sector fully mobilize the scientific research sector to actively participate in and use of grids technology sharing through establishing cooperation mechanism, to achieve resources sharing platform for agricultural research. All the research resources planed overall, unified the production standards, system construction, uniform sharing mechanism to avoid duplication of resources, to avoid duplication of investment funds, put an end to the wasted phenomenon, and help to improve the quality of construction resources, enhance the degree of resource sharing [8].

\section{Grids-based Agriculture Research Resource Sharing System}

There are a large number of heterogeneous in agriculture research resource system. The resources heterogeneity exists in the platform layer, system layer and semantic layer. It is currently a serious problem to integrate existing heterogeneous agriculture research resource library and to provide a large-scale resource sharing platform, grids can solve it, the agriculture research resource sharing platform structure shown in Fig.1.

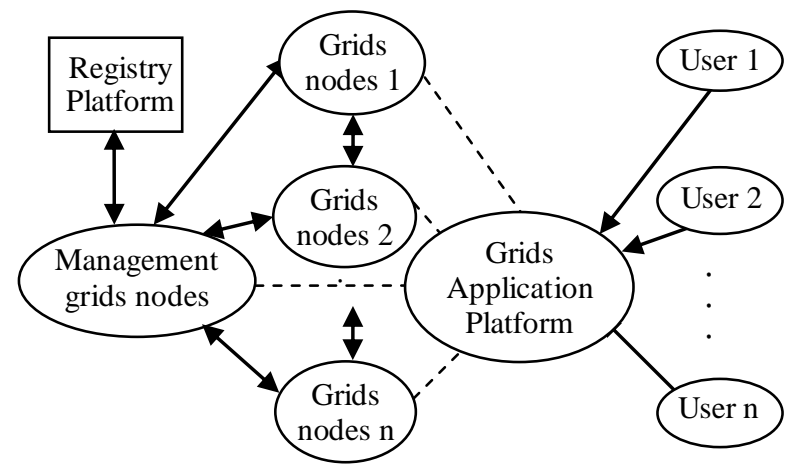

Figure 1. Agriculture network resource sharing platform based on Grids

\section{A. Grids Node}

Grids nodes is the most fundamental part in the system, they not only implement the grids function, but also includes the grids resources and grids services. The available resources and equipment in various agricultural areas can be used as grids nodes are linked to the shared platform.

\section{B. Registration platform}

Its function is to provide users with a wide range of agricultural services available to the grids. In grids, it can be layered by multiple service registration platforms. The client or learner can find all registered teaching services in grids by registration service platform. For the teaching system developers, can found grids services in the registry Platform, directly embedded into the development of teaching software which; and for learners, through the grids platform can execute learning activities.

\section{Grids platform}

It is the user interface to access grids services, also can be viewed as Web page to provide grids services. Grids users get into the grids system through grids application platform, to obtain the required services.

\section{User}

Users are persons to use the grids services in the share-based resource library grids system, the user can not only use grids resources and enjoy grids services, but also can build their own applications using the grids resources and services. Users must register through the grids portal page to access the agricultural resources. 
Persons on Each grids node who design and develop their own digital resources uploaded to the uniform interface, the user on the other nodes automatically called GridFTP, the agricultural resources files are synchronized to the other nodes, The agricultural resources are automatically distributed and synchronized.

Use grids resource sharing capabilities to construct the integrated and virtual interface. So the users focus on the use of resources only, without considering the system architecture, technical route, location of resources, and so on.

An ideal grids system should be built on all hardware and software in use, providing fully transparent computing environment, integrating a number of heterogeneous resources into a virtual computing resources to the user. In this paper, OGSA (Open Grids Services Architecture) is used. OGSA is an important criterion for Global Grids Forum4 proposal, is called the next generation of the grids structure, it has become the default standard of actual development [9]. Guided by OGSA service, we built a network of agricultural resource sharing system based on OGSA shown in Fig. 2. The framework is divided into four layers: application layer, middle layer, service layer and resource layer.

\begin{tabular}{|c|c|c|c|}
\hline App 1 & App 2 & $\mathrm{App} \mathrm{n}^{\mathrm{App}}$ & $\begin{array}{l}\text { plication } \\
\text { layer }\end{array}$ \\
\hline Grid Service Interface & \begin{tabular}{l|l} 
hterface & Grid S \\
\end{tabular} & \multicolumn{2}{|c|}{\begin{tabular}{|l|l} 
Grid Services Management & Middle \\
layer
\end{tabular}} \\
\hline $\begin{array}{c}\begin{array}{c}\text { Service } \\
\text { container1 }\end{array} \\
\end{array}$ & $\begin{array}{c}\begin{array}{c}\text { Service } \\
\text { container } 2\end{array} \\
\end{array}$ & $\begin{array}{c}\text { Service } \\
\text { container } \mathrm{n}\end{array}$ & \multirow{2}{*}{$\begin{array}{l}\text { Service } \\
\text { layer }\end{array}$} \\
\hline $\begin{array}{c}\text { Grid } \\
\text { Service 1 } \\
\end{array}$ & $\begin{array}{c}\text { Grid } \\
\text { Service 2 } \\
\end{array}$ & $\begin{array}{c}\text { Grid } \\
\text { Service } \mathrm{n} \\
\end{array}$ & \\
\hline $\begin{array}{c}\text { Resource } \\
\text { model } 1\end{array}$ & $\begin{array}{l}\text { Resource } \\
\text { model } 2\end{array}$ & $\begin{array}{c}\text { Resource } \\
\text { model } \mathrm{n}\end{array}$ & \multirow{2}{*}{$\begin{array}{c}\text { Resource } \\
\text { layer }\end{array}$} \\
\hline $\begin{array}{l}\text { Resource } \\
\text { database } 1\end{array}$ & $\begin{array}{l}\text { Resource } \\
\text { database } 2\end{array}$ & $\begin{array}{l}\text { Resource } \\
\text { database } \mathrm{n}\end{array}$ & \\
\hline
\end{tabular}

Figure 2. agricultural research resource sharing model

Application layer included all application program involved, they need a variety of services respectively, it provide interactive action through the application software between resource services and supporting software, the application program which know the services clearly can get the services directly through Grids Service Interface, the application which do not know the services clearly can search grids service through grids services management and discovery to get the grids services.

Middle layer consists of services resources and applications software between middle layer and application layer, the grids service interface and the grids service management and discovery. Grids service interface interact with the application interface, the application get the known grids services via a standard HTTP+SOAP (Simple Object Access Protocol) request; grids service management and discovery combined Grids Service set to register into the middle layer, made it easy to find the Grids Services. Grids service management and discovery technology will used to inform service registry and UDDI (Universal Description Discovery and Integration) standard to complete the registration of each Grids Service.

Service layer resources, including resource layer Grids Service, operate and manage each grids service container, make the grids service management and discovery in the middle layer can find these services. Resource layer include the agricultural network resources and model library, the resources basic information, the facilities dynamic information.

Based on the above system, the implementations of the system are described below: 


\section{A. Distributed resource in all areas sharing}

The services management and discovery in the middle layer of the grids system not only let the service developers in all the country to release their services resources, but also provide all applications with grids services.

\section{B. The various applications of Application layer execute in parallel}

In this system, each application, in addition to the necessary interaction, implement their own program independent, so each application have the higher parallelism degree. This has greatly enhanced the agricultural resource device utilization and the applications execution efficiency.

\section{Good performance with heterogeneous}

In this system, a variety of network resource may be inconsistent to access system; the programming language may be inconsistent. But the upper grids services are consistent through the grids Service of the service layer and the Apache. In OGSA, the service interface description is implemented by a platform independent description language WSDL, service interface function is implemented by the cross-platform java programming language; they achieve cross-platform development. As long as the services compliance with OGSA specifications, no matter what is the host environment, the code which implement the services are portable to other execution environments directly.

\section{Scalability}

Scalability must be considered with the increase of the number of agricultural resources. In this system, as long as the service compliance with the OGSA specification, added to the grids services management and discovery of the middle layer through the Apache server of the service layer. They can be applied and searched by the applications, implement entry and exit freely.

\section{Exploration of the Agricultural Resource Sharing Model}

Agricultural research resources should plan overall, Implement step by step. Take the Ministry of agriculture Technical Specifications and other standards as a guide, combined with academic, professional, and curriculum development resources for building standard features. Develop them according to the actual needs of agricultural development. Actively introducing and transforming quality resources at home and abroad, implement the second development, but also cooperate with the domestic large-scale agricultural resources developer. Resources must meet the needs of agricultural development, closely around the agriculture, professional, technology to build them, and consider the application of practical application. Construction agricultural resources must be planed and managed overall.

Commercial operation is conducive to promote the rational flow of social resources. Resource builders, business, education and training institutions help to optimize agricultural resources, to play their respective advantages, agricultural resource operators will become bigger and stronger, while risk tolerance will be increased. Commercial operation will improve effectiveness. On the one hand to meet market needs, to train more high-quality research personnel for the community; on the other hand the value of agricultural resources will increase, this can greatly improve the social and economic benefits of agricultural resources, and enhance the vitality and sustainable development of agricultural resources.

In the construction of agricultural resources, incentive mechanisms motivate the participants to build a resource, they are important means and methods to motivate the creativity and arouse their enthusiasm toward the desired goal, and they also are the basic mechanisms in effective building agricultural resources. Market operations mechanisms establishment are incentive to agricultural resources' property protection and reproduction, to arouse the enthusiasm of resource developer, is a major Effective operation mode in industrial environment of knowledge economy and information Industry. Agriculture administration, business administration and other departments take some guidance and standardization measures in the birth, run and developing, to avoid market monopoly, fraud and Chaos, make agricultural Resources Construction and Application develop well. 


\section{Conclusion}

Agricultural research Resources is the foundation for agricultural research and technical means, it is a measure of a comprehensive scientific research capacity of an organizations. Agricultural research resource sharing can play important role in teaching, research and the social services, and excavate the potential of existing research resources, reduce and avoid duplication of purchase, effectively alleviate the shortage of funds and improve the utilization of large-scale precision resources. Share of agricultural resources regardless of time and geographical constraints, the user can determine the actual experimental test content and the progress, which can best meet the different needs of users, to provide a good environment for network resource sharing to multi-sectoral, multi-industry, multi-unit, multi-research unit to carry out collaborative research and development

Grid-based agricultural research sharing method aimed at sharing equipment of resources, increased agricultural research and resource utilization; be able to break through the various administrative independence of the units or departments, conduct resources and experimental data sharing, strengthen cooperation and collaboration, improve agricultural scientific research; enhance and link social enterprise or unit, so that resource owners who need to interact with the resource services, To make use efficiency and economic benefits of agricultural research resources.

\section{References}

[1] Jin H, ChinaGrid: Making grids computing a reality, Int'l Collaboration and Cross-Fertilization(ICADL 2004), Berlin, 2004, pp.13-24

[2] Chen Xin, Kang Li, Grid construction and agricultural information service network exploration, Agriculture Network Information, 2005, vol.01, pp. 20-25, 39

[3] European Data Grids, Datagrid.http://eu-datagrid.web.eern.eh/eu-datagrid, 2009

[4] Ian Foster, Carl Kesselman, Grids computing, Jin Hai translation, 2nd. Beijing: Electronic Industry Press, 2004, pp176-187

[5] Nemeth Z, Sunderam V, Characterizing grids: Attributes, definitions, and formalisms, Journal of Grids Computing, Vol. 1, Issue 1, 2003, pp.9-23

[6] Foster I, Kesselman C, Nick J, The Physiology of the Grid: An Open Grid Service Architecture for Distributed Systems Integration. http://www.globus.org/research/papers/ogsa.pdf, 2008

[7] Ruay-Shiung Chang, Jih-Sheng Chang, Shin-Yi Lin, Job scheduling and data replication on data grids, Future Generation Computer Systems, vol.23, Issue 7, 2007, pp.289-295

[8] Zhang Junfeng, Service-based grid system of agricultural research resources, Graduate School of Chinese Academy of Agricultural Sciences, 2007

[9] Du Zhihui, Chen Yu, Liu Peng, service-centered grid architecture OGS A, Computer Science, 2003, vol.30 (7), pp. 26-29 